\title{
The Lurcher Cerebellar Mutant Phenotype Is Not Expressed on a staggerer Mutant Background
}

\author{
Anne Messer, Bonnie Eisenberg, ${ }^{a}$ and Julie Plummer \\ Wadsworth Center for Laboratories and Research, New York State Department of Health, Albany, New York 12201-0509 \\ and Neuroscience Program, Department of Biomedical Sciences, School of Public Health, SUNY at Albany, Albany, \\ New York 12201-0509
}

\begin{abstract}
The hierarchy of the various processes responsible for the development of the complex, elaborated Purkinje cell can be examined by taking advantage of a series of spontaneous mutations that affect cerebellar development in the mouse. This study uses double mutants containing genes for two separate hereditary cerebellar mutations that have been shown to act intrinsically in Purkinje cells in order to investigate the time course and modes of action of these mutations. Lurcher mice show $100 \%$ degeneration of Purkinje cells, starting during the second postnatal week, while staggerer mice show reduced numbers of Purkinje cells in a distinctive mediolateral distribution from the time of birth, with the remainder grossly stunted. When these mutations are combined genetically, mice shown by progeny tests to harbor both staggerer and Lurcher genotypes exhibit staggerer-like behavior and overall cerebellar morphology; they also do not lose $100 \%$ of their Purkinje cells, as Lurcher mutants would otherwise do. Instead, they show a characteristic staggerercerebellar pathology. We conclude that the intrinsic action of the staggerer gene in Purkinje cells occurs earlier in development than do effects of the Lurcher gene, and that the action of the staggerer gene prevents Purkinje cells from acquiring the characteristics required for the cytotoxic action of the Lurcher gene.
\end{abstract}

The development of a complex neuron must involve precise orchestration of gene expression along a genetically predetermined pathway, with some contribution from external signals in most cells. Neurological mutants that interfere with normal development in a consistent way provide a useful set of tools to study this process in vivo. There are several mutations in the mouse that are known to lead to abnormal cerebella (Green, 1989). Two of these, staggerer $(s g)$ and Lurcher $(L c)$, have a primary target of gene action in Purkinje cells (Herrup and Mullen, 1979a; Wetts and Herrup, 1982). The former, a recessive mutation on chromosome 9 , is characterized by ataxic

\footnotetext{
Received Oct. 15, 1990; revised Mar. 21, 1991; accepted Mar. 29, 1991.

We wish to thank Carl Eriole for histology, Dr. Abigail Snyder-Keller and Ms. Catherine Clarke for assistance with immunocytochemistry, Dr. Sylvia Christakos for the initial calbindin antibody, and Dr. Karl Herrup for helpful discussions. This work was supported by NIH Grant NS17633 to A.M.

Correspondence should be addressed to Anne Messer, Ph.D., Wadsworth Center for Laboratories and Research, New York State Department of Health, P.O. Box 509, Albany, New York 12201-0509.

a Present address: School of Public Health, Yale University, New Haven, CT 06520.

Copyright (C) 1991 Society for Neuroscience $0270-6474 / 91 / 112295-08 \$ 03.00 / 0$
}

symptoms from postnatal day (P) 8 . Histologically, the cerebellum shows reduced numbers of Purkinje cells (25-50\%, varying in severity along a mediolateral axis) from before birth (Herrup and Mullen, 1979b), with residual Purkinje cells severely stunted and lacking tertiary dendritic spines (Sotelo and Changeux, 1974; Hirano and Dembitzer, 1975; Landis and Sidman, 1978). Lurcher, a dominant mutation on chromosome 6, expressed in $L c /+$, does not show symptoms until about P12, though there are morphological changes in Purkinje cells of the most developmentally advanced portions of the cerebellum as early as P6 (Heckroth et al., 1990). These cells begin to die during the second postnatal week, progressing to $100 \%$ loss by 5 weeks (Caddy and Biscoe, 1979). Both mutants show effects on granule cells, including almost complete loss of these cells with time, secondary to the Purkinje cell abnormalities (Sotelo and Changeux, 1974; Sotelo, 1975; Landis and Sidman, 1978; Caddy and Biscoe, 1979).

There are several lines of evidence that the gene defect in $s g$ / $s g$ is expressed earlier in development than the gene defect in $L c /+$. As noted above, symptoms and gross morphological changes appear somewhat sooner in $s g / s g$. Functionally, the small, stunted $s g / s g$ Purkinje cells appear not to signal effectively either the onset or the cessation of neuronal cell proliferation in the external granule cell layer (Landis and Sidman, 1978; Messer et al., 1981; Yoon, 1976). Granule cell proliferation in $L C /+$ mice is only slightly affected (Caddy and Biscoe, 1979; Wetts and Herrup, 1983), and there is evidence that $L c /+$ Purkinje cells can interact with granule cells prior to their degeneration (Vogel et al., 1989). Staggerer mice cannot mediate thyroid hormone signals for either cerebellar thymidine kinase induction or neurotransmitter amino acid maturation (Messer, 1988; Messer et al., 1988); $L c /+$ mutants appear particularly sensitive to excess thyroid hormone, with alterations of taurine, glutamic acid, aspartic acid, and GABA levels that are most consistent with an acceleration of the degenerative process (Messer et al., 1989). As assayed by Northern blot or in situ hybridization histochemistry, $s g / s g$ Purkinje cells do not contain detectable levels of calmodulin mRNAs (which are strongly induced shortly after birth), while $L c /+$ Purkinje cells apparently contain normal levels of these mRNAs until the cells disappear (Messer et al., 1990).

These data do not, however, exclude the possibility that a very early intrinsic change in Lurcher Purkinje cells leads to their degeneration. In order to determine whether the apparent block in the differentiation of the $s g / s g$ Purkinje cells could prevent the degenerative process from occurring in $L c /+$ Purkinje cells, double mutants containing both the $s g / s g$ and $L c /+$ 
genotypes were bred. Results suggest that the $L c /+$ genetic program cannot be expressed in $s g / s g$ Purkinje cells.

\section{Materials and Methods}

Colonies. The staggerer colony is maintained here by brother-sister mating of C57BL sg/dse (dilute/short-ear) heterozygotes originally obtained from the Jackson Laboratory several years ago. If kept in a pathogen-free environment and given high-fat food (Purina) on the cage floor plus long water spouts for drinking after weaning, approximately half of the $\mathrm{sg} / \mathrm{sg}$ females will breed for one or two litters. Foster care is sometimes necessary to assure healthy offspring. Males carrying $\mathrm{sg} / \mathrm{sg}$ on this inbred background cannot hold a mount to mate. The Lurcher colony was established here on a BALB/cByJ background as previously described (Messer et al., 1989). Both males and females are fertile and mate. Mice are checked daily for births. Animal procedures have been approved by the Institutional Animal Care and Use Committee.

Experimental crosses. The breeding scheme is shown in Figure 1. A C57BL $s g / s g$ female was mated to an $L c /+$ male, and a male Fl with $\mathrm{Lc} /+$ symptoms was backcrossed with a second C $57 \mathrm{BL} \mathrm{sg} / \mathrm{sg}$ female from a different litter. Of the progeny from this cross, hybrid males exhibiting mutant symptoms were progeny tested by mating to both an inbred $s g / s g$ female and a wild-type (BALB/cByJ) female. If the former gave litters that were $100 \% \mathrm{sg} / \mathrm{sg}$, and the latter gave litters containing some $L c /+$ mice (determined by both behavioral and histological criteria), the male was considered to be a proven $s g / s g: L c /+$ mouse.

Behavioral testing. Mutant $s g / s g$ mice are identifiable at $8 \mathrm{~d}$ of age by splaying of the hindlimbs and ataxic gait. By $10 \mathrm{~d}$, a tendency to fall over sideways when attempting to run is apparent. By $14 \mathrm{~d}$, they are obviously smaller than their normal littermates, and they show a clear staggering gait. The lifespan is reduced, but with extra care, as noted above, more than half survive into adulthood. Mutant $L c /+$ mice show mild gait impairment and clutching of the hindlimbs beginning at about P12. As they age, they lurch from side to side, but rarely fall. Most $L c /+$ mice are of normal size and live at least $1 \mathrm{yr}$.

Histopathology. All mice were adults ( 5 months old for the double mutants; $2-3$ months old for $s g / s g$ and $L c /+$ ) killed by transcardial perfusion of $4 \%$ paraformaldehyde under deep ether anesthesia. Brains were embedded in paraffin and sectioned at $10 \mu \mathrm{m}$, as previously described (Messer and Flaherty, 1986), with both hematoxylin and eosin (H\&E) staining and thionin staining for most cases.

Immunocytochemistry. Antibody to calbindin, the $28-\mathrm{kDa}$ calciumbinding protein that has been shown within the cerebellum to localize exclusively to Purkinje cells (Gona et al., 1986; Christakos et al., 1987) was used to verify the identity of the mutant Purkinje cells. Initial experiments were done with rabbit antisera from S. Christakos (Christakos et al., 1987), which was used at a primary antibody concentration of 1:1000. Later work was done using a monoclonal anti-calbindin obtained from Sigma Chemical Company (1:200), HRP-conjugated goat anti-mouse second antibody (Cappel; 1:50), and a nickel-intensified diaminobenzidine substrate.

\section{Results}

\section{Genetics and behaviors}

The breeding scheme and genotypes of selected progeny are shown in Figure 1. Approximately half the F1 mice produced from the original $s g / s g \times L c /+$ matings had an $L c /+$ gait abnormality; there seemed to be no effects of the obligate staggerer heterozygosity in combination with $L C /+$. There also did not seem to be any effects of the BALB/C57BL hybrid vigor on the age of onset or on the severity of the Lurcher symptoms. Fifteen surviving progeny of two litters from matings of an $\mathrm{F} 1 \mathrm{LC} /+$ : $s g /+$ male by an inbred (C57BL) $s g / s g$ female showed the following: (1) five mice with abnormally small size and $s g / s g$-like behavior (splaying of hindlimbs by $8-9 \mathrm{~d}$ and a tendency to fall when running by $10 \mathrm{~d}$, though they could get up and move more easily after falling than the inbred $s g / s g$ mice); (2) four mice with apparent $L C /+$ phenotypes (mild gait impairment beginning at about P12, with continued lurching as they age; no apparent differences between these and inbred $L c /+$ mice); and (3) six mice that were apparently normal.
These numbers are within statistical limits for either the Lurcher or the staggerer phenotype predominating, or for a mixed phenotype in the $L c /+: s g / s g$ double mutants (Green, 1981). Difficulties in obtaining large numbers of progeny from inbred $s g / s g$ females made direct analysis of a small number of mice the most feasible option for continuing studies. The probable $\mathrm{sg} / \mathrm{sg}$ mice from these F1/backcross litters seemed somewhat stronger and larger than our inbred $\mathrm{sg} / \mathrm{sg}$ mice; this is presumably due to a generalized hybrid vigor. Such males were also (quite fortunately) able to mate.

In order to test whether any of the mice that appeared to be staggerers were double mutants carrying both $s g / s g$ and a cryptic Lurcher gene, candidate males were mated simultaneously to C57BL sg/sg females and to $+1+$ BALB females. (Only the potential doublc-mutant males could be progeny tested at this stage, because it was not possible to get any inbred $\mathrm{sg} / \mathrm{sg}$ males to mate with possible double-mutant females. It would also have been difficult to breed enough litters from double-mutant females to confirm both genotypes.) For two of the males tested, $100 \%$ of progeny of crosses with inbred $\mathrm{sg} / \mathrm{sg}$ showed the $\mathrm{stag}$ gerer symptoms (P8-9 onset, staggering, and falling), while several of the progeny of matings with $+/+$ females showed $\mathrm{Lc} /+$ symptoms ( $>$ P12 onset, lurching gait).

\section{Histopathology}

The $s g / s g$ nature of the double mutant and the expected cerebellar pathologies of the mice from the progeny test were confirmed histologically in adults. Examination of the full adult cerebellum, at low microscopic power, illustrates characteristic changes in the sizes and shapes of the two inbred mutants versus the wild-type. As described previously (reviewed in Green, 1989), the $s g / s g$ cerebella are noticaby smaller and less foliated than the $L c /+$ cerebella, which are considerably smaller than wildtype (Fig. $2 A-C$, sagittal). The genetically identified double mutant (genealogy: Fig. 1, case 1), shown in coronal section (Fig. $2 D$ ), clearly demonstrates an overall $s g / s g$ morphology. Mice from the progeny test (genealogy: Fig. 1, cases 2, 3) have cerebellar shapes that match their symptoms, that is, no Purkinje cell layer but modest foliation for $L C /+$ (Fig. $2 E$ ) and disrupted lamination and very little foliation for $s g / s g$ (Fig. $2 F$ ).

At higher magnification, it is possible to observe individual Purkinje cells. With H\&E staining, wild-type Purkinje cells are large, slightly oval, pale-staining cells with dark nuclei, arranged in a single layer along the boundary of the internal granule cell layer (Fig. 3A). There is no sign of any such large cells in the $L C /+$ cerebellum (Fig. $3 B$ ). The $s g / s g$ cerebellum, near the midline, shows a population of medium-to-large pale cells with dark nuclei, scattered in a wide, multirow layer (Fig. 3C). The double mutant also shows many of these cells (Fig. 3D), while the $L \mathrm{c} /+$ mouse fathered by this double mutant mated to a $+1+$ female shows none (Fig. $3 E$ ). Progeny of this same double mutant with an $s g / s g$ mother again show the medium-to-large pale cells (Fig. $3 F)$.

\section{Immunocytochemistry}

In order to verify that the medium-to-large neurons seen in the double mutant actually are Purkinje cells, antibody to the Purkinje cell-specific protein calbindin was employed. In agreement with our previous (unpublished) studies and some recently reported by Celio (1990), Purkinje cells from an inbred $\mathrm{sg} / \mathrm{sg}$ cerebellum show clear labeling with anti-calbindin (Fig. $4 A$ ). There are absolutely no labeled cells in a section from an inbred 


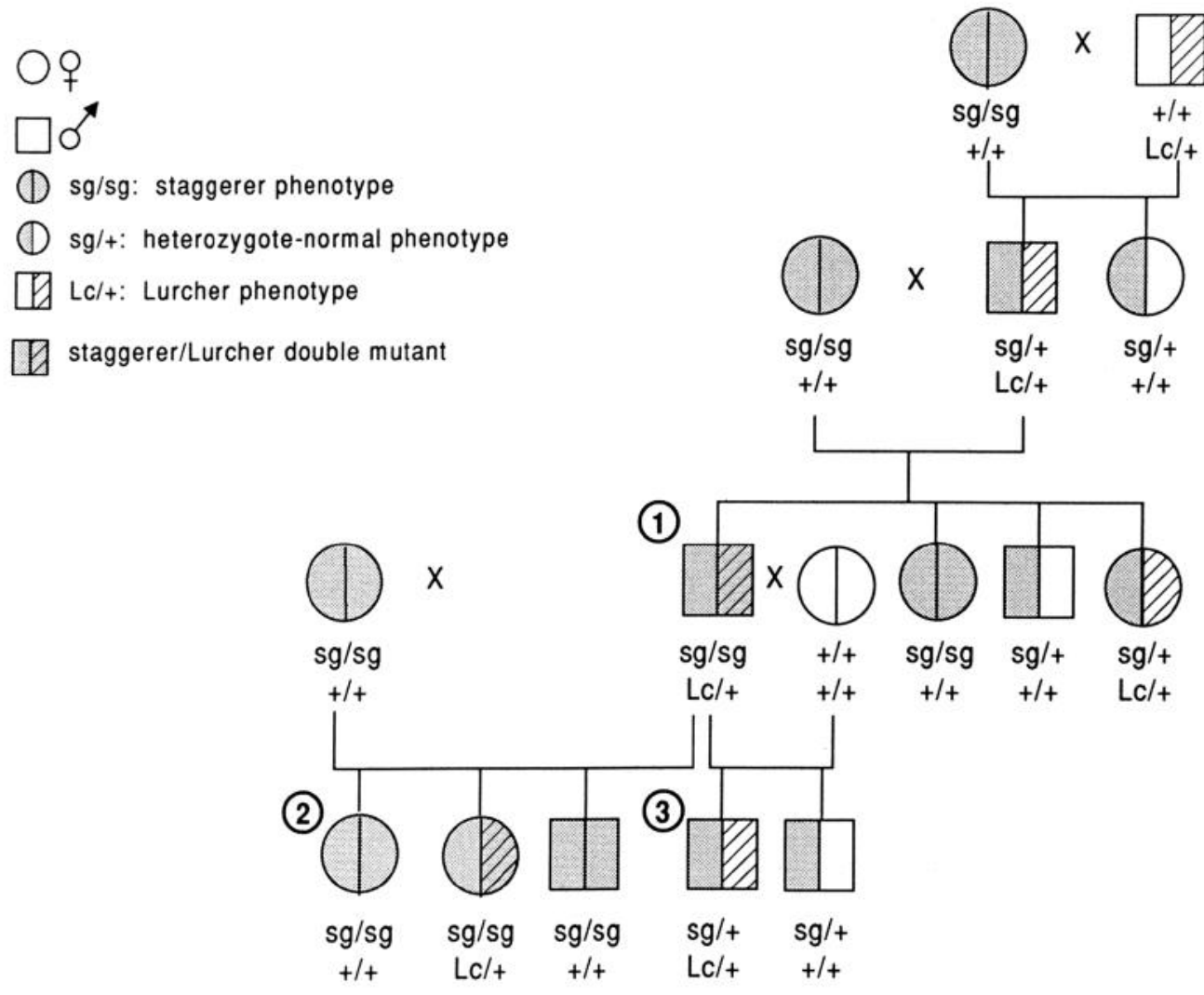

Figure 1. Genealogy of double mutants, showing genotypes of selected progeny. Circled numbers to the left of individual figures are their case numbers, referred to in the remaining figure captions and in the text. The staggerer gene is designated by shading (the entire symbol for homozygotes; half the symbol for heterozygotes); the Lurcher gene is designated by hatching. adult $L c /+$ cerebellum (Fig. $4 B$ ). Sections from two different areas of the double mutant (Fig. $4 C, D$ ) show many Purkinje cells scattered in a broad band, confirming the observations seen with the H\&E stain.

\section{Discussion}

This study shows that a mutant that has been verified genetically as carrying both $s g / s g$ and $L c /+$ genes expresses only the $s g / s g$ phenotype for behavior and adult cerebellar histopathology. Because both genes act intrinsically in Purkinje cells (Herrup and Mullen, 1979a; Wetts and Herrup, 1982), the results with the double mutant strongly suggest that the full Lurcher phenotype cannot be expressed within a Purkinje cell that is genetically $s g$ / sg.

The data clearly show that almost all of the Purkinje cells normally present in $s g / s g$ escape the degenerative fate that the Lurcher gene they carry would otherwise dictate. The possibility that the effect of the Lurcher gene causes degeneration of just a few of the Purkinje cells in double-mutant animals cannot be definitely excluded, because the loss of a small additional percentage of the already-reduced number of $\mathrm{sg} / \mathrm{sg}$ Purkinje cells would be hard to identify without doing comprehensive cell counts in a large group of $s g / s g: L c /+$ and $s g / s g:+/+$ mice from the same litters. However, the modest amelioration of the symptoms seen here in double mutants versus the inbred $\mathrm{sg} / \mathrm{sg}$ seems more likely to be due to hybrid vigor than to a loss of additional abnormal Purkinje cells due to a modifying effect of the Lurcher gene. The $s g / s g:+/+$ littermates of the double mutants (defined as mice with $s g / s g$ symptoms that had $0 \mathrm{Lc} /+$ progeny out of 10 or more when mated to wild-type) were clinically indistinguishable from $s g / s g: L c /+$, and the severity of the neurological phenotype of $s g / s g$ is known to be affected by the genetic background of the mice (Sidman et al., 1962).

The question of mechanism really must be asked at two levels: initial effects of the gene, and the death of the Purkinje cells. These may both be the direct effect of a single gene product, or the cell death may be the last link in a chain of events started by an aberrant protein several steps earlier. Caddy and Biscoe (1979) describe alterations in the dendritic arbors, including multiple primary dendrites, at P14, as well as spherical mitochondria in some of the cells that are presumably in the process of degenerating at this same time. Heckroth et al. (1990) describe dendritic changes in the midvermal $L c /+$ Purkinje cells as early as P6, with persistence of perisomatic nests of climbing fibers, retention of somatic spines, and a failure of basket cell axon terminals to appear. As an intrinsically acting dominant gene, Lurcher could possibly code for a structural protein or integrated membrane protein such as a receptor tyrosine kinase (Pawson and Bernstein, 1990) that could by itself be responsible for both an early change in dendritic morphology (and trophism?) and the eventual death of the cells. These double-mutant experiments address only the question of whether the end point of the phenotypic change, Purkinje cell loss, can occur in cells that also carry $s g / s g$.

In a series of studies of the fine structure of staggerer/reeler $(r l)$ double mutants, Yoon (1977b) observed that the staggerer Purkinje cell soma and dendrites could not be distinguished from double-mutant Purkinje cells on a morphological basis. Because subsequent studies have proven that the staggerer gene acts intrinsically in Purkinje cells, while the reeler gene acts extrinsically (reviewed in Green, 1989), this result suggests that the $s g / s g$ Purkinje cell genetic program cannot be affected by 
Figure 2. Photomicrographs of adult cerebellar sections stained with $H \& E$, showing overall shape and layering for wild-type, inbred single mutants, and cases 1, 2, and 3 from Figure 1. $A,+/+$ (BALB), sagittal. $B, L c /+$, sagittal. $C$, $s g / s g$, sagittal. $D$, Case 1, coronal (sg/ $s g: L c /+) . E$, Case 3, coronal $(s g /+: L c l$ $+)$. F, Case 2, coronal (sg/sg.+/+). Scale bars, $250 \mu \mathrm{m}$.
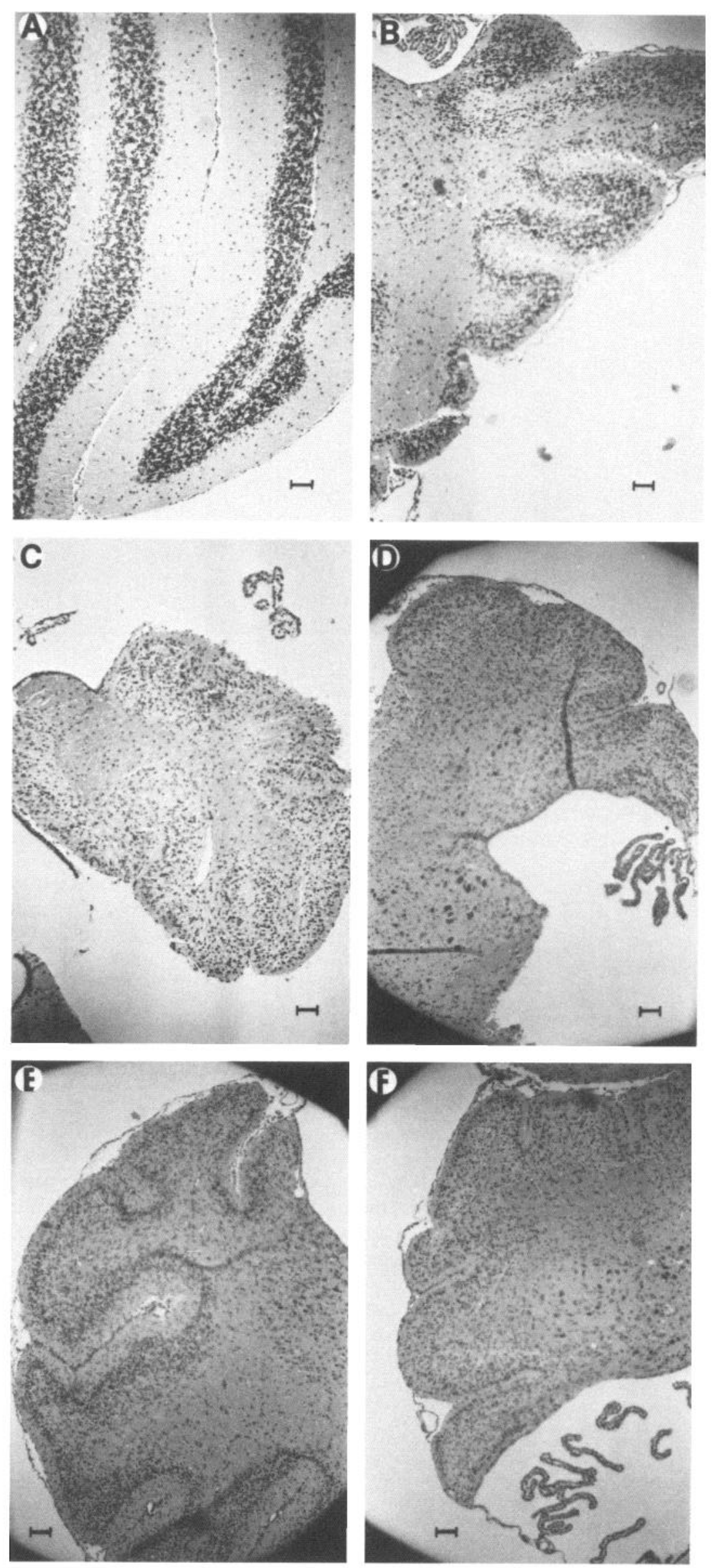

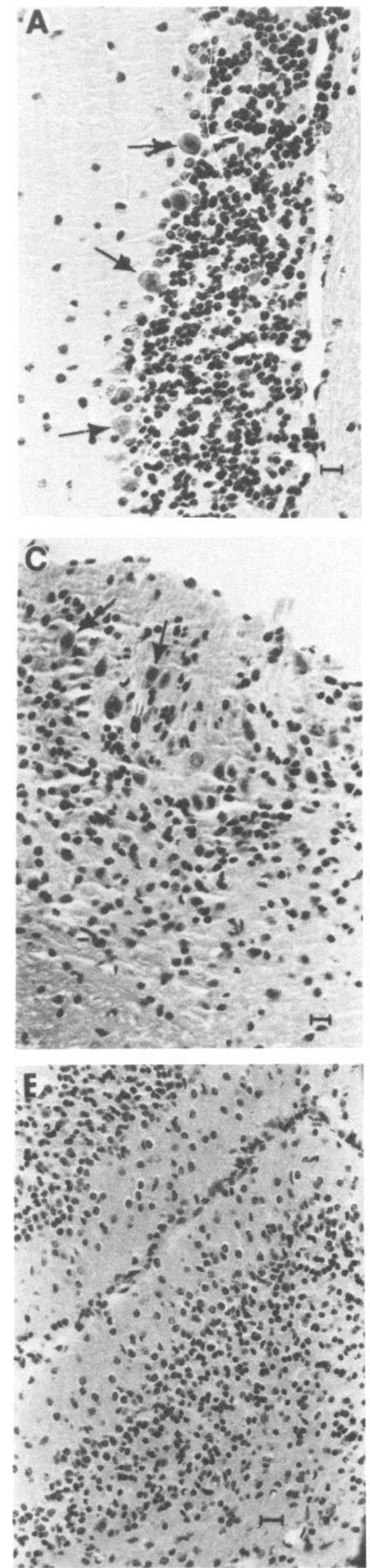
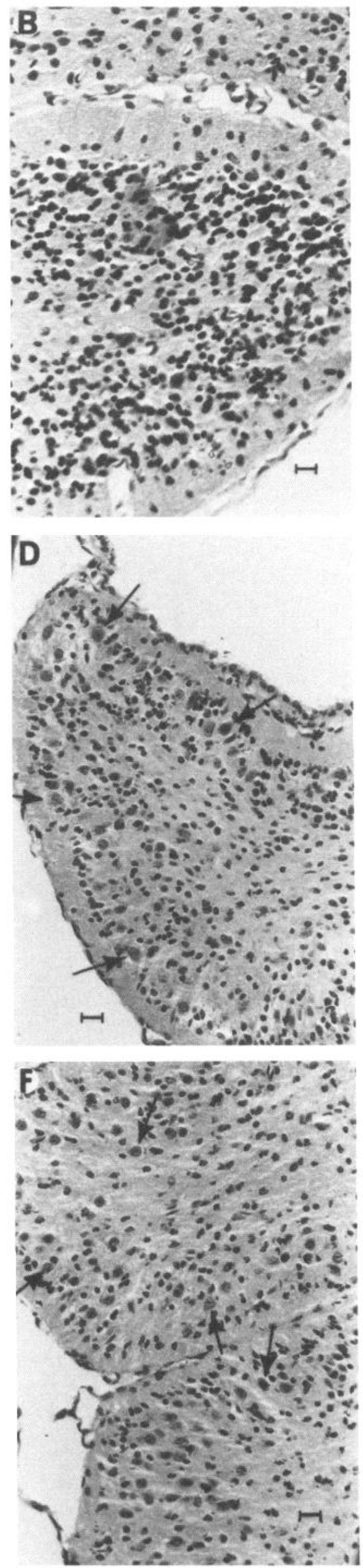

Figure 3. Photomicrographs of the Figure 2 cerebellar sections at higher magnification, showing individual Purkinje cells and their groupings. $A,+/+$ (BALB), sagittal. $B, L c /+$, sagittal. $C$, $s g / s g$, sagittal. $D$, Case 1 , coronal $(s g /$ sg: $L c /+)$. E, Case 3, coronal (sg/+: $L c /$ + ). $F$, Case 2, coronal (sg/sg:+/+). Scale bars, $25 \mu \mathrm{m}$. 

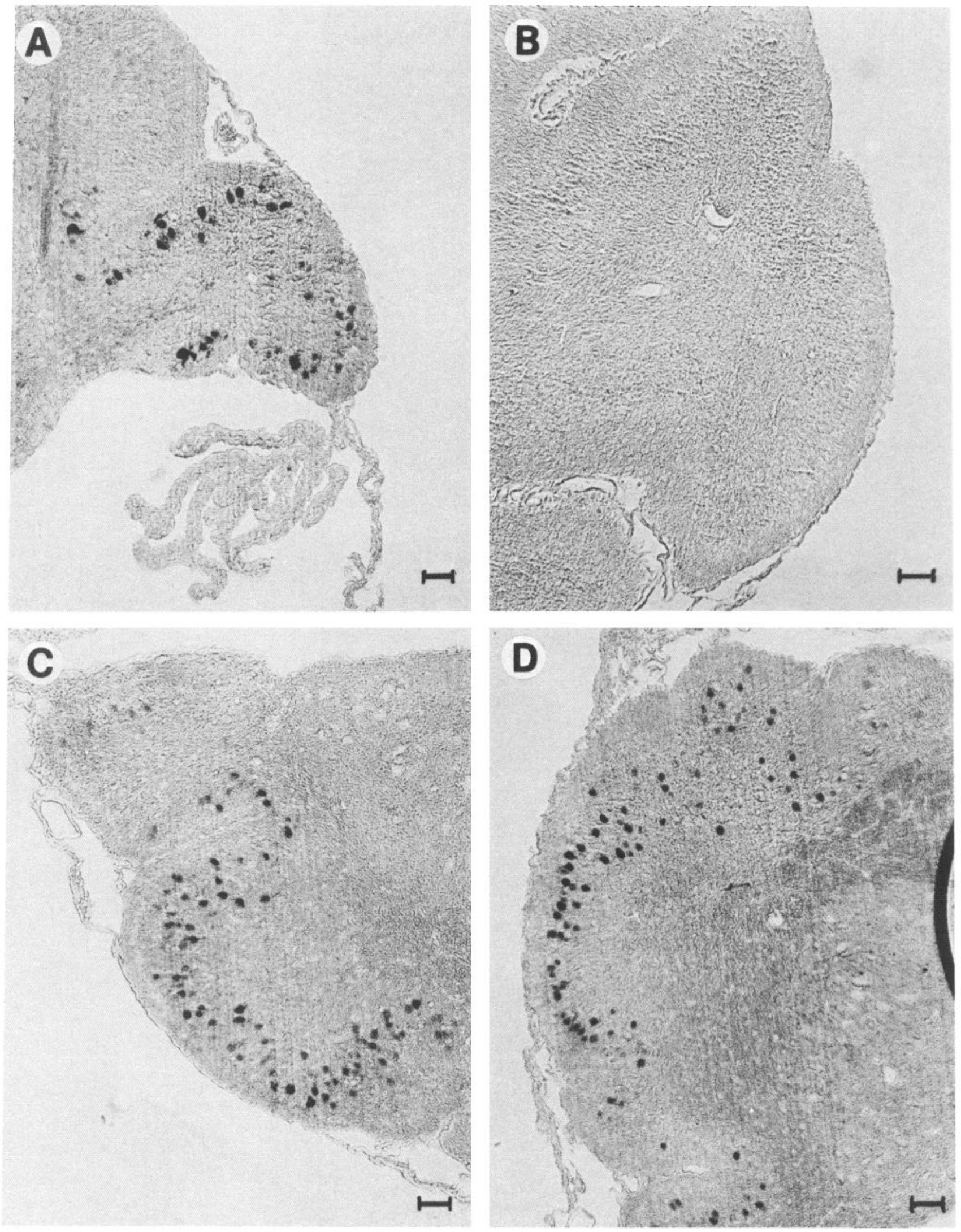

Figure 4. Photomicrographs of cerebellar coronal sections reacted with anti-calbindin antibody, visualized using HRP. A, Case 2 (sg/sg: $+/+)$. B, Case $3(s g /+: L c /+)$. $C$ and $D$, Case $1(s g / s g: L c /+)$. Scale bars, $50 \mu \mathrm{m}$. 
the external influence of the reeler gene. This is quite similar to the results reported here for $\mathrm{Lc} / \mathrm{sg}$ double mutants. However, unlike this study, the external granule cell layer of the $s g / r l$ double mutant seems to show an additive effect of the two mutations, because it completely disappears by P15 (Yoon, 1977a). The genetic interaction reported for two different forms of retinal degeneration (Sanyal and Hawkins, 1981), in which the absence of rhodopsin and the outer segment slows the normal course of degeneration of the $r l$ phenotype, is closer to what we have found.

Interference with the Lurcher gene action in $s g / s g$ Purkinje cells could be occurring in several ways: (1) The Lurcher gene may be part of a set of genes acting in more mature Purkinje cells that are never transcribed if earlier genes are not turned on first. The prenatally induced genes for glutamic acid decarboxylase and calbindin appear to be, by in situ hybridization studies of mRNA, expressed at relatively normal levels both in Lurcher Purkinje cells up until the time they disappear (Wuenschell et al., 1990) and in $s g / s g$ Purkinje cells (G. Franz, A. Messer, and A. J. Tobin, unpublished observations). However, we have shown that $m$ RNAs for the three calmodulin genes are undetectable in $s g / s g$ Purkinje cells, but are apparently normal in Lurcher Purkinje cells for as long as they are present (Messer et al., 1990). In wild-type mouse cerebellum, these mRNAs undergo a tremendous increase in level during the first week after birth, particularly between P0 and P4. Some other mRNAs normally induced after P10 also seem not to be transcribed in $s g / s g$ Purkinje cells (M. L. Vallano, G. Feussner, and A. Messer, unpublished observations; J. Oberdick, personal communication). Therefore, $s g / s g$, which interferes in some way with transcripts for early postnatal genes, may be preventing the transcription of later-onset genes, including the Lurcher gene itself. (2) The Lurcher gene product may be present, but cannot be integrated into a membrane or other compartment because of the prior effect of the $\mathrm{sg} / \mathrm{sg}$ gene. As noted above, an intrinsic, dominant mode of gene action implies that Lurcher could be an enzymatic membrane protein or a structural gene. Such proteins may be particularly susceptible to this type of integrational interference. For example, if the Lurcher gene product must be integrated into the membrane of the tertiary dendritic spine in order to kill the Purkinje cell, it could not function in $s g / s g$ Purkinje cells that lack such spines. [The studies of Caddy and Biscoe (1979) show what do appear to be such spines in P14 $l c l+$ Purkinje cells, though it is somewhat difficult to distinguish secondary from tertiary spines.] This hypothesis neither requires nor excludes the possibility that an early dendritic change effected by the Lurcher gene, which might be functionally and morphologically transparent in adults, could occur in $s g / s g: L c /$ + Purkinje cells; however, it does require that the actual mechanism of the cell death be dependent upon integration into more mature parts of the dendrite. (3) The Lurcher gene product may be in the right place, but its abnormal function may require the proper functioning of other cellular processes that have been disrupted by $s g / s g$. For example, the Lurcher gene product may be toxic only in the presence of some other intact dendritic action (e.g., a specific calcium current) that is lacking in the $s g$ / $s g$ Purkinje cell. This hypothesis is also neutral with respect to cell death and earlier $L c /+$ changes.

A distinction among these hypotheses is very difficult if progeny testing is required to confirm the genotypes of the double mutants, because the optimal developmental studies are then impossible. Potentially, the double mutants can be recognized much earlier if there are DNA polymorphisms that can identify the chromosome carrying the Lurcher gene. Unfortunately, none of the genes closely linked to Lurcher ( $I g K$ and ornithine decarboxylase- 5 being the two most likely candidates) have been reported to show any polymorphism between a BALB allele, which would designate the chromosomal region as coming from Lurcher, and a C57BL allele, which would identify that region of chromosome 6 as coming from the $\mathrm{sg} / \mathrm{sg}$ parent (Elliott, 1989). As further DNA markers are added to the mouse map, this situation may improve. A closer examination of the morphology of the Purkinje cell dendrites and their afferents in the adult animals might reveal some differences between $s g / s g:+1+$ and $s g / s g: L c /+$ that would support the hypotheses that postulate interrelated and sequential, but distinct, processes responsible for early dendritic changes and for cell death.

The major objective of these experiments is to provide a means of dissecting Purkinje cell differentiation in vivo. They do suggest that, whatever the process killing Lurcher Purkinje cells, the complete expression of the genetic program is dependent upon the correct development of the Purkinje cell up to a certain point. The data are also compatible with a hypothesis that Purkinje cell development may proceed by sequentially expressing groups of genes for "early," "intermediate," and "late" functions, similar to those that have been described for a variety of other cells and unicellular organisms. However, in order to utilize this information fully, it will be necessary to know what the actual mechanisms of action of both the Lurcher and the staggerer genes are. Hopefully, this work has also made some progress in that direction, by further restricting the possibilities of when and where in development to look for the abnormal action of these genes.

\section{References}

Caddy KWT, Biscoe TJ (1979) Structural and quantitative studies on the normal $\mathrm{C} 3 \mathrm{H}$ and Lurcher mutant mouse. Philos Trans 287:167201.

Celio MR (1990) Calbindin D-28k and parvalbumin in the rat nervous system. Neuroscience 35:375-475.

Christakos S, Rhoten WB, Feldman SC (1987) Rat calbindin D28K: purification, quantitation, immunocytochemical localization, and comparative aspects. Methods Enzymol 139:534-551.

Elliott RW (1989) DNA polymorphisms. In: Genetic variants and strains of the laboratory mouse, Vol 2 (Lyon MF, Searle AG, eds), pp 537-573. Oxford: Oxford UP.

Gona AG, Pendurthi TK, Al-Rabiai S, Gona O, Christakos S (1986) Immunocytochemical localization and immunological characterization of vitamin D-dependent calcium-binding protein in the bullfrog cerebellum. Brain Behav Evol 29:176-183.

Green EL (1981) Genetics and probability in animal breeding experiments. New York: Oxford UP.

Green MC (1989) Catalog of mutant genes and polymorphic loci. In: Genetic variants and strains of the laboratory mouse, Vol 2 (Lyon MF, Searle AG, eds), pp 12-403. Oxford: Oxford UP.

Heckroth JA, Goldowitz D, Eisenman LM (1990) Olivocerebellar fiber maturation in normal and lurcher mutant mice: defective development in lurcher. J Comp Neurol 291:415-430.

Herrup K, Mullen RJ (1979a) staggerer chimeras: intrinsic nature of Purkinje cell defects and implications for normal cerebellar development. Brain Res 178:443-457.

Herrup K, Mullen RJ (1979b) Regional variation and absence of large neurons in the cerebellum of the staggerer mouse. Dev Brain Res 172:1-12.

Hirano A, Dembitzer HM (1975) The fine structure of staggerer cerebellum. J Neuropathol Exp Neurol 34:1-11.

Landis DMD, Sidman RL (1978) Electron microscopic analysis of postnatal histogenesis in the cerebellar cortex of staggerer mutant mice. J Comp Neurol 179:831-864.

Messer A (1988) Thyroxine injections do not cause premature induction of thymidine kinase in $s g / s g$ mice. J Neurochem 51:888-891. 
Messer A, Flaherty L (1986) Autosomal dominance in a late-onset motor neuron disease in the mouse. J Neurogenet 3:345-355.

Messer A, Savage M, Carter TP (1981) Thymidine kinase activity is reduced in the developing staggerer cerebellum. J Neurochem 37: $1610-1612$.

Messer A, Eisenberg B, Martin DL (1988) Role of timing and cell interactions in cerebellar development. In: Disorders of the developing nervous system: changing views on their origins, diagnoses and treatment (Swann JW, Messer A, eds), pp 111-123. New York: Liss.

Messer A, Eisenberg B, Martin DL (1989) Effects of mild hyperthyroidism on levels of amino acids in the developing Lurcher cerebellum. J Neurogenet 5:77-85.

Messer A, Plummer-Siegard J, Eisenberg B (1990) staggerer mutant mouse Purkinje cells do not contain detectable calmodulin mRNA. J Neurochem 55:293-302.

Pawson T, Bernstein A (1990) Receptor tyrosine kinases: genetic evidence for their role in Drosophila and mouse development. Trends Genet 6:350-357.

Sanyal S, Hawkins RK (1981) Genetic interaction in the retinal degeneration of mice. Exp Eye Res 33:213-222.

Sidman RL, Lane PW, Dickie MM (1962) staggerer, a new mutation in the mouse affecting the cerebellum. Science 137:610-612.

Sotelo C (1975) Dendritic abnormalities of Purkinje cells in the cerebellum of neurologic mutant mice (weaver and staggerer). In: Advances in neurology, Vol 22 (Frentzberg GW, ed), pp 335-351. New York: Raven.

Sotelo C, Changeux JP (1974) Transsynaptic degeneration 'en cascade' in the cerebellar cortex of staggerer mutant micc. Brain Res 67:519526.

Vogel MW, Sunter K, Herrup K (1989) Numerical matching between granule and Purkinje cells in Lurcher chimeric mice: a hypothesis for the trophic rescue of granule cells from target-related cell death. J Neurosci 9:3454-3462

Wetts R, Herrup K (1982) Interaction of granule, Purkinje, and inferior olivary neurons in Lurcher chimeric mice. I. Qualitative studies. J Embryol Exp Morphol 68:87-98.

Wetts R, Herrup K (1983) Direct correlation between Purkinje and granule cell numbers in the cerebella of Lurcher chimeras and wildtype mice. Dev Brain Res 10:41-47.

Wuenschell CW, Messer A, Tobin AJ (1990) Lurcher Purkinje cells express glutamic acid decarboxylase and calbindin mRNAs. J Neurosci Res, in press.

Yoon CH (1976) Pleiotropic effect of the staggerer gene. Brain Res 109:206-215.

Yoon CH (1977a) Fine structure of the cerebellum of "staggererreeler," a double mutant of mice affected by staggerer and reeler conditions. I. The premature disappearance of the external granular layer and ensuing cerebellar disorganization. J Neuropathol Exp Neurol 36:413-426.

Yoon CH (1977b) Fine structure of the cerebellum of "staggererreeler," a double mutant of mice affected by the staggerer and reeler conditions. II. Purkinje cell anomalies. J Neuropathol Exp Neurol 36: $427-439$. 